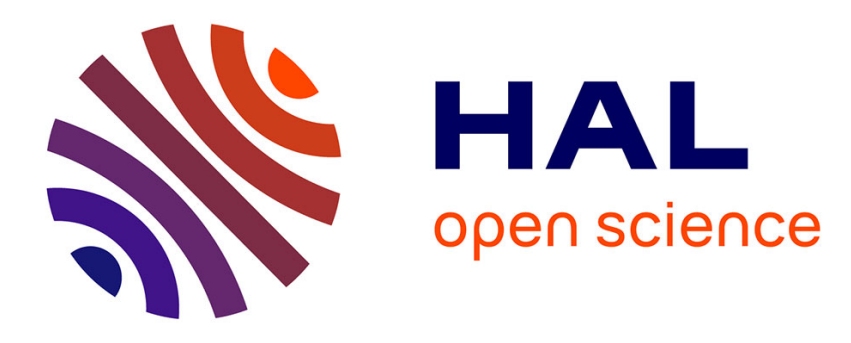

\title{
Computation of interatomic Green functions for transition metals using continued fraction techniques
}

\author{
G. Tréglia, A. Bieber
}

\section{To cite this version:}

G. Tréglia, A. Bieber. Computation of interatomic Green functions for transition metals using continued fraction techniques. Journal de Physique, 1984, 45 (2), pp.283-290. 10.1051/jphys:01984004502028300 . jpa-00209754

\section{HAL Id: jpa-00209754 https://hal.science/jpa-00209754}

Submitted on 1 Jan 1984

HAL is a multi-disciplinary open access archive for the deposit and dissemination of scientific research documents, whether they are published or not. The documents may come from teaching and research institutions in France or abroad, or from public or private research centers.
L'archive ouverte pluridisciplinaire HAL, est destinée au dépôt et à la diffusion de documents scientifiques de niveau recherche, publiés ou non, émanant des établissements d'enseignement et de recherche français ou étrangers, des laboratoires publics ou privés. 
Classification

Physics Abstracts

$71.10-71.20-71.25 \mathrm{C}-71.25 \mathrm{~F}$

\title{
Computation of interatomic Green functions for transition metals using continued fraction techniques
}

\author{
G. Tréglia and A. Bieber (*) \\ Laboratoire de Physique des Solides, Bât. 510, Université Paris-Sud, 91405 Orsay, France \\ (*) CRM-CNRS, 6, rue Boussingault, 67083 Strasbourg Cedex, France et LMSES (LA 306), \\ 4, rue Blaise Pascal, 67070 Strasbourg Cedex, France
}

(Reçu le 27 juin 1983, accepté le 3 octobre 1983)

\begin{abstract}
Résumé. - Nous calculons ici des fonctions de Green interatomiques, dans le cas de métaux de transition, par une technique d'espace réel : la méthode de récursion. Nous comparons nos résultats, pour des métaux CFC, à ceux que l'on peut tirer des calculs de bande par une technique d'espace des $k:$ la méthode des tétraèdres. L'accord est aussi bon que dans le cas des fonctions de Green intra-atomiques usuelles. Nous montrons de plus, dans ce dernier cas, que les densités d'états reconstruites sous forme de fractions continues et celles déduites des calculs de bande sont quasiment indiscernables, pour peu qu'on ait calculé un nombre suffisamment grand de coefficients exacts.
\end{abstract}

\begin{abstract}
Interatomic Green functions are computed here for transition metals using a real space technique, namely the recursion method. The results for the case of the FCC structure are compared to those derived from band structure calculations using a $k$-space technique, namely the tetrahedron method. We show here that the agreement between both calculations is as good as for usual intra-atomic Green functions. In this last case, we show that, provided that a sufficient number of exact coefficients are calculated, the densities of states reconstructed by continued fraction techniques are almost indistinguishable from those obtained from band structure calculations.
\end{abstract}

\section{Introduction.}

The study of a great variety of problems requires the computation of interatomic Green functions : this is the case in particular, each time that a coupling between electrons located on different sites is involved. On the other hand, the knowledge of these functions in real space is often convenient, if not always necessary. This occurs not only for problems in which the lattice periodicity is lost (alloys, defects, surfaces and adsorbates, ...) but also when we are interested in the local environment of an atom, even in a perfect lattice. Then, the Bloch functions in $k$-space which are uniformly extended over the whole crystal are indeed completely inadequate. In this last case, it is possible to get the physical properties of interest by a summation in $k$-space, but it is at the expense of the physical understanding of what is done.

Usual $k$-space techniques, which are very useful in many cases, are not very convenient for computing real space interatomic Green functions, especially when they are required in the whole complex plane : a major drawback is that these methods yield only the imaginary part of the Green function, the real part being calculated by a Hilbert transform.
We report here a real space technique which gives an analytic expansion of these real space Green functions : they are written as algebraic summations of continued fractions [1-3]. Up to now, this method has only been applied to tight binding $d$ bands and is thus particularly well suited for transition metals. Its main advantages are the following :

i) the interatomic Green functions $\tilde{G}^{R}(z)$ are analytic in the whole complex plane whereas they have to be calculated numerically for each value of the energy $z$ by $k$-space methods;

ii) both real and imaginary parts of $\tilde{G}^{R}(z)$ are directly obtained, which avoids the Hilbert transform which is in general numerically ill-conditioned;

iii) for a given lattice structure and for each neighbour distance $R$ a set of coefficients completely characterizes the Green function (at least for standard electronic parameters);

iv) working in real space enables us to get a physical insight into the approximations that we can use - or not - (" local " approximations [4], summations restricted to a given shell of neighbours [5], ...).

We do not claim here that we present a new method since the formalism of the recursion method is well 
known [1-3], but our aim is twofold. First we point out that this recursion method allows us to reproduce all the fine structures of a density of states provided that we calculate a sufficiently large number of exact coefficients; the densities so-calculated are then almost indistinguishable from those derived from band structure calculations. Second, we show that, despite being based on completely different computations, the interatomic Green functions obtained both through the recursion method and through the so called tetrahedron method [6] are in as good an agreement with one another as the corresponding intra-atomic ones. This implies that we can use the recursion method for calculating interatomic Green functions with the same confidence as the $k$-space methods.

\section{The continued fraction technique.}

We first briefly summarize the recursion method $[1,2]$ for calculating intra-atomic Green functions, discussing the main problems and showing new results. Then, we discuss its generalization to interatomic Green functions. In both cases, results are compared to those derived from band structure calculations through the tetrahedron method [6].

\subsection{INTRA-ATOMIC GREEN FUNCTIONS.}

2.1.1 Method. - These intra-atomic Green functions are very useful since their imaginary parts give the densities of states. Their expansion in terms of continued fractions is well-known :

$$
\begin{aligned}
G_{\lambda \lambda}^{00}(z)=\frac{1}{z-a_{1}^{\lambda \lambda}}-\frac{b_{1}^{\lambda \lambda}}{\mid z-a_{2}^{\lambda \lambda}}-\cdots- \\
-\frac{b_{n}^{\lambda \lambda}}{\mid z-a_{n+1}^{\lambda \lambda}}-\cdots
\end{aligned}
$$

where :

$G_{\lambda \lambda}^{00}(z)=\left\langle 0, \lambda\left|(z-H)^{-1}\right| 0, \lambda\right\rangle$ and $z=E+i 0$

$|0, \lambda\rangle$ is the atomic orbital of type $\lambda$ (d band symmetry : $\lambda=x y, y z, z x, x^{2}-y^{2}, 3 z^{2}-r^{2}$ ) centred at site 0 , and $H$ is the usual tight binding Hamiltonian. The coefficients of the continued fraction, $a_{n}^{\lambda \lambda}$ and $b_{n}^{\lambda \lambda}$, are closely related to the moments of the density of states and are obtained either from these moments [7] (calculated by counting paths in the lattice) or directly within the recursion method [1, 2]. We have chosen the latter method since it avoids the transformation of moments into coefficients, which is not well conditioned from a numerical point of view.

The aim of the recursion method is to build, from the atomic orbital basis set, a new basis in which the Hamiltonian is tridiagonal (the first vector of this new basis being chosen equal to the atomic orbital on which the local density of states is calculated). Then, if we denote by $a_{n}^{\lambda \lambda}$ and $\sqrt{b_{n}^{\lambda \lambda}}$ the diagonal and offdiagonal elements of $H$ in this new basis, it is easy to show that the intra-atomic Green function at site 0 with symmetry $\lambda$ (Eq. 2) can be written as (Eq. 1). In such a calculation, the only parameters are obviously the lattice structure and a finite set of hopping integrals fitted to band structure calculations within interpolation schemes [8]. In practice, we have to introduce three hopping integrals $\operatorname{dd} \sigma\left(R_{0}\right)$, $\mathrm{dd} \pi\left(R_{0}\right)$ and $\operatorname{dd} \lambda\left(R_{0}\right)$, for a given neighbouring distance $R_{0}$ and to assume a certain law for the variation of the hopping integrals with distance : a decreasing exponential law $\left(\operatorname{dd} \beta(R)=\operatorname{dd} \beta\left(R_{0}\right) \exp \left(-q\left(\frac{R}{R_{0}}-1\right)\right)\right.$ with $3 \leqslant q R_{0} \leqslant 5$ ) has been shown to be realistic by Ducastelle [9]. This law introduces a natural cut-off on distances, which means that only a finite number of hopping integrals has to be considered in the Hamiltonian : for instance, only first neighbours are needed for the FCC structure whereas the second ones are also required for the $\mathrm{BCC}$ one. Furthermore, this set of three integrals can be reduced to one for a given lattice by noting that their ratios do not vary very much along a transition series [9] : $\mathrm{dd} \pi / \mathrm{dd} \sigma \simeq-0.5$ and $\operatorname{dd} \delta / \operatorname{dd} \sigma \simeq 0$; thus, they all can be expressed in terms of $\operatorname{dd} \sigma$ for instance. Therefore, everything is defined except for the energy units : it means that we shall have for a given lattice, an archetypical density of states, the bandwidth of which will be fitted to that of the metal of interest.

It is obvious from the recursion formulae [1] that the construction of the new basis requires the formation of a cluster growing rapidly with the number of exact coefficients that is needed. Then the main difficulty comes from the fact that, due to computer storage limitations, only a finite and rather small number of exact coefficients, $N$, is available to reconstruct the densities of states (in general, for simple structures $N=11-13$ ). The problem is then to terminate this finite continued fraction. Several solutions exist :

i) the continued fraction can be truncated at the last exact level $\left(b_{N}=0\right)$; the density of states is then made of $N$ delta functions that can be broadened by adding a small imaginary part to the energy;

ii) the cluster built for $N$ exact coefficients can be used to calculate more coefficients $\left(a_{n>N}, b_{n>N}\right)$ but the latter coefficients characterize the finite cluster and not the whole crystal; in this case, $b_{n} \rightarrow 0$ for a finite value of $n$;

iii) if there is no gap in the density of states, the coefficients $a_{n}$ and $b_{n}$ converge towards asymptotic values $a_{\infty}$ and $b_{\infty}$ (which are related to the centre and the width of the $\mathrm{d}$ band respectively) when $n \rightarrow \infty$. The usual termination is then to replace $a_{n}$ and $b_{n}$ by these asymptotic values for $n>N$. $a_{\infty}$ and $b_{\infty}$ can be guessed for a sufficiently large number of exact coefficients; they can be obtained exactly in terms of $\operatorname{dd} \sigma, \operatorname{dd} \pi$ and $\operatorname{dd} \delta$ for simple structures;

iv) if a gap exists in the density of states (alloys, ...), these coefficients present undamped oscillations : then 
they are periodic functions of $n$. A method has been recently reported to characterize these asymptotic oscillations which allows accurate densities of states to be obtained even in this case [10]. This can be seen by comparing the results of Turchi et al. [10] for $\mathrm{Cu}_{3} \mathrm{Au}$ type alloys to those obtained by Koenig [11] using a LMTO calculation.

2.1.2 Application to FCC metals. - The densities of states of pure metals are in general reconstructed with about 11 or 13 couples of exact coefficients (clusters of about 5000 atoms), the continued fraction being terminated by replacing other coefficients by their asymptotic values. However, at least to our knowledge, no investigation concerning the influence of higher order coefficients on the details of the density of states has been reported yet. Therefore, we have computed the density of states of an archetypical FCC density of states, using Fletcher hopping parameters [12] :

i) within the tetrahedron method (see insert of Fig. 1)

ii) using the recursion method with 11 and 18 couples of exact coefficients (see Fig. 1); notice that the latter calculation (18 coefficients) requires a cluster of 21127 atoms, which was tractable only due to the very large storage capacity of the computer (Amdahl V7) of the CIRCE computer centre (CNRS-Orsay).

The overall agreement between the densities of states obtained within the tetrahedron method and the recursion one with 11 coefficients (usual FCC curve) is of course good. However, the $k$-space calculation exhibits more peaks : in particular, one sees three well defined peaks instead of two broadened structures in the direct space calculation, in the lower part of the band. These features significantly change when the

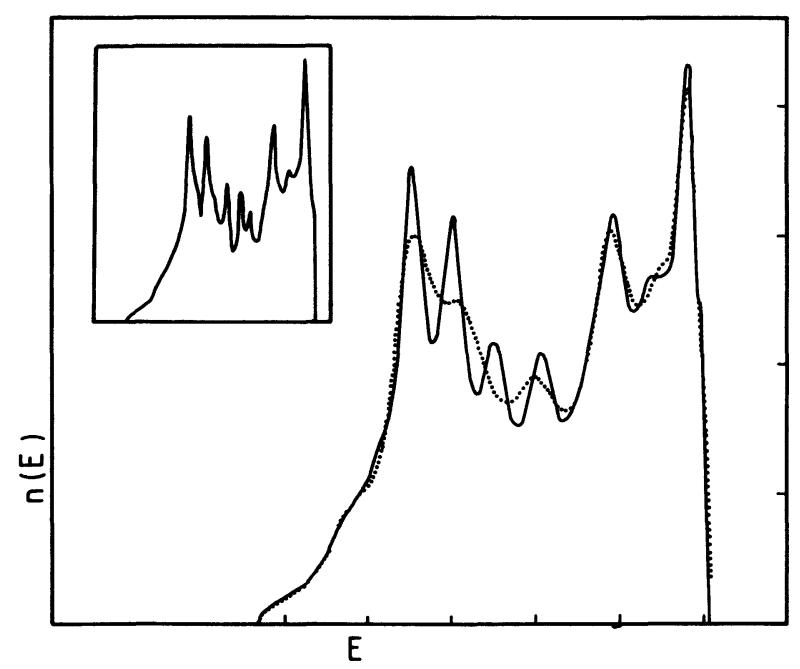

Fig. 1. - Density of d states of an FCC metal, reconstructed with 11 (dotted line) and 18 (full line) couples of exact coefficients computed using the recursion method. In the insert we show the same density of states calculated using the tetrahedron method. number of exact coefficients increases from 11 to 18 couples : in the latter case, all the previous structures are now clearly resolved in position as well as in intensity. Both calculations (recursion and tetrahedron) are then almost indistinguishable. This is an appealing result, even if the details of the densities of states do not play an important rôle for computing integrated quantities such as energies [13] : actually, it invalidates the common idea that the knowledge of about 20 or 25 exact moments (10-13 couples of exact coefficients) is sufficient to exhibit all the details of a density of states and that moment or recursion methods can only yield smooth structures which cannot be compared in detail with the sharp peaks derived from band structure calculations. The same behaviour is observed for alloys in the split band regime [11, 13]. It is of interest when comparing theoretical curves to those deduced from angular resolved photoemission experiments.

\subsection{INTERATOMIC GREEN FUNCTIONS.}

2.2.1 Method. - The technique described in $\$ 2.1$.1 can be generalized to the case of interatomic Green functions $\widetilde{G}_{\lambda \mu}^{R}(z)$ by using a simple mathematical transformation [3] :

$$
\begin{aligned}
& \tilde{G}_{\lambda \mu}^{R}(z)=\left\langle 0, \lambda\left|(z-H)^{-1}\right| R, \mu\right\rangle= \\
& \quad=G_{\lambda \mu}^{0 R}-\left(G_{\lambda \lambda}^{00}+G_{\mu \mu}^{R R}\right) / 2
\end{aligned}
$$

where $G_{\lambda \mu}^{0 R}$ is the intra-atomic Green function, calculated as previously explained, for the pseudo siteorbital : $|r, v\rangle=(|0, \lambda\rangle+|R, \mu\rangle) / \sqrt{2}$; then :

$$
G_{\lambda \mu}^{0 R}=G_{v v}^{r r}=\left\langle r, v\left|(z-H)^{-1}\right| r, v\right\rangle .
$$

Note that $G_{\mu \mu}^{00}=G_{\mu \mu}^{R R}$ if the lattice sites 0 and $R$ are equivalent.

Therefore, $\widetilde{G}^{R}$ is obtained as an algebraic summation of intra-atomic Green functions (i.e. a summation of continued fractions); this enables us to retain all the advantages previously described of the continued fraction technique (analyticity in the whole complex plane, real and imaginary parts obtained simultaneously, ...).

Then, for a given lattice and for given $R, \lambda, \mu, \tilde{G}_{\lambda \mu}^{R}$ will be completely characterized by the set of coefficients of the intra-atomic elements $G_{\lambda \mu}^{0 R}$, since the coefficients of $G_{\lambda \lambda}^{00}$ and $G_{\mu \mu}^{R R}$ have been computed once for all.

From a computational point of view, it is necessary to be very careful with respect to the numerical accuracy, particularly for distant neighbours (in some problems, $\widetilde{G}_{\lambda \mu}^{\boldsymbol{R}}$ are required up to the sixth neighbour). This accuracy may be checked in view of the sum rules that Green functions have to satisfy. Actually, it can be shown that, when $R$ increases, an increasing number of first moments of $\tilde{G}_{\lambda \mu}^{R}$ must vanish $[5,13]$; hence, $\tilde{G}^{R}(E)$ must exhibit a minimum number of zeros when $E$ goes from the bottom to the top of the band. For example, for the FCC lattice, $\widetilde{G}^{1}$ has at 
least one zero whereas $\widetilde{G}^{2}, \widetilde{G}^{3}$ and $\widetilde{G}^{4}$ show at least two zeros (for the sake of simplicity, we denote by $R=1,2,3$ and 4 the first, second, third and fourth neighbouring distances.

2.2.2 Application to FCC metals. - Using these results, we have computed the interatomic Green functions within both tetrahedron and recursion (11 couples of exact coefficients) methods. As an example, the imaginary part of $\widetilde{G}_{x y, x y}^{R}(O x$ and $O y$ axes join first neighbours) up to the fourth neighbour is shown in figure 2. The overall agreement is at least as good as for intra-atomic Green functions for the same number of exact coefficients (the same agreement is found for the real part). Furthermore, one can note that small discrepancies occur for the same energy values as for intra-atomic Green functions (lower part of the band) so that increasing the number of exact coefficients will probably improve this agreement and resolve the sharp peaks. Nevertheless, these $\widetilde{G}^{\boldsymbol{R}}$ are in most cases used in integrated quantities for which such details are not relevant; therefore, we shall restrict ourselves here to $N=11$.

It is clear from figure 2 that the amplitude of $\tilde{G}^{R}$ decreases roughly with the neighbouring distance $R$. In fact, this is not strictly true as can be noticed by comparing $R=3$ and $R=4$. Actually, the relevant parameter here is not exactly the distance between two sites but rather the nature of the path leading from one to the other (number of hops, geometry of the path, etc.). For example here, both 3rd and 4th neighbours are reached with two hops but the path to the 4th neigh-

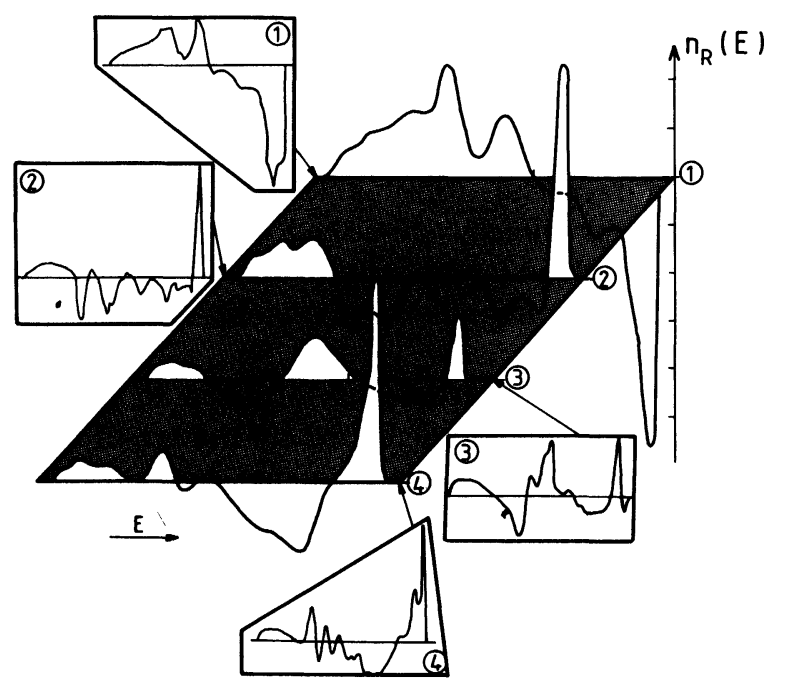

Fig. 2. - Interatomic « densities » : $n_{R}(E)=-\frac{\operatorname{Im}}{\pi} \tilde{G}_{11}^{R}(E)$; computed up to the 4 th neighbour $(R=1,2,3,4)$ using the recursion method (11 couples of exact coefficients). $|1\rangle$ is the $|x y\rangle$ orbital, in our system of axes $(\mathrm{O} x$ and $\mathrm{O} y$ join first neighbours), (see table I). In the inserts we show the corresponding results of calculations using the tetrahedron method. bour is a straight line whereas it is a broken line for the 3rd one and, as shown by Ducastelle (unpublished results) and Hirai and Kanamori [15], the contribution of the first class of paths is more important than that of the latter. However, such effects have to be taken into account only for neighbours that are reached with the same number of hops and they cannot affect the overall decreasing behaviour of $\tilde{G}^{R}$ with the distance $R$. We have carried out our calculation up to the sixth neighbour : the results show that $\widetilde{G}^{2}, \widetilde{G}^{3}$ and $\widetilde{G}^{4}$ are of similar importance and much smaller than $\widetilde{G}^{1}$, while $\widetilde{G}^{5}$ and $\widetilde{G}^{6}$ are found to be completely negligible. Finally, let us note that the required minimum number of zeros is well reproduced by figure 2 .

\section{Examples of applications.}

As mentioned in the introduction, the $\tilde{G}^{R}$ can be involved in various physical problems. Let us just mention two of them that we have been able to treat only by applying the previous method.

The first one is the systematic study of the stabilities of the ordered structures occurring in transition metal alloys. Actually, such an investigation requires the computation of chemical and magnetic cluster interactions $[5,16]$ that are expressed in terms of $\widetilde{G}^{\boldsymbol{R}}$ and have to be calculated accurately up to the 4th neighbour. This for example, is needed in order to compare relative stabilities of ordered structures such as those of $\mathrm{Cu}_{3} \mathrm{Au}$ and $\mathrm{Al}_{3} \mathrm{Ti}$ types [13]. Let us just note that similar calculations, performed in some particular cases by $k$-space techniques (M. Hennion, private communication), are in good agreement with ours.

The second problem is the study of the influence of electronic correlations, in the band limit, within a perturbation method on the Hubbard model. The second-order term in $U / W$ ( $U$ : Coulomb integral, $W: \mathrm{d}$ band width) of the total ground state energy involves a tedious multiple integration in $k$-space that can be avoided by coming back into real space. This energy is then written [4] as a sum over neighbouring distances of products of $\tilde{G}^{R}$ over a denominator. Our direct space technique allows us to show that the local term $(R=0)$ accounts for $98 \%$ of the sum and then to neglect other terms, which considerably facilitates the following calculations.

Finally, let us mention here the calculations of local and non local spin susceptibilities in transition metals, performed by Terakura et al. [17] and Hirai [18] : their results are completely consistent with ours.

To sum up, we think that our comparison between $k$ - and direct space calculations justifies the use of the latter for a great variety of problems. Moreover, in order to perform a systematic study in a very simple way, we have assumed here constant ratios of Slater parameters $(\mathrm{dd} \sigma, \mathrm{dd} \pi, \mathrm{dd} \delta)$ for all FCC metals. This is of course not strictly true, but small deviations from these values only lead to very small effects, the general trends being determined by geometrical effects so 
that such a simple scheme can be used as a good approximation. Hence, we have a set of « universal " coefficients for a given lattice, that can be suited to each particular transition metal by just fitting its bandwidth. Therefore, in view of the large number of physical problems in which such tables of coefficients could be useful without resorting to any tedious calculations, we have included them in the appendix for the FCC structure.

\section{Acknowledgments.}

The authors would like to express their sincere thanks to Drs. F. Ducastelle, F. Gautier and P. Turchi for innumerable and enlightening discussions about the moment problem and the recursion method. They are also very grateful to Dr. P. Leonard who provided them with his tetrahedron routine. Finally, one of the authors (A.B.) is deeply indebted to Mr. M. Gendner and Mr. G. Weill for their efficient computational assistance at the CNRS Computer Center of Strasbourg Cronenbourg.

\section{Appendix.}

In this appendix, we give all information required to reconstruct the interatomic Green functions, up to the 4th neighbour, in an FCC lattice (similar tables can be obtained from the authors for BCC).

i) All the $\tilde{G}_{\lambda \mu}^{R}$ are given in terms of a few independent ones, using symmetry properties, in table I. In this table, $R=1,2,3,4$ corresponds to 1 st, 2 nd, 3 rd and 4th neighbours respectively; $a$ is the distance between first neighbours and the three orthogonal axes are such that $O x$ and $O y$ join first neighbours in the (100) plane. The orbitals $x y, y z, z x, x^{2}-y^{2}$ and $3 z^{2}-r^{2}$ are labelled 1, 2, 3, 4 and 5 respectively.

ii) These independent $\tilde{G}_{\lambda \mu}^{R}$ are obtained from equation 3 by computing $G_{\lambda \lambda}^{00}, G_{\mu \mu}^{R R}=G_{\mu \mu}^{00}$ and $G_{\lambda \mu}^{0 R}$ with the coefficients of table II. These coefficients have been calculated for a bandwidth: $W_{0}=2.7 \mathrm{eV}$ (Fletcher [12] parameters); therefore, for a given FCC metal characterized by its bandwidth $W$ (fitted to band structure calculations) for a given volume, the coefficients $a_{n}$ and $b_{n}$ of table II have just to be multiplied by $\left(W / W_{0}\right)$ and $\left(W / W_{0}\right)^{2}$ respectively. The continued fractions are terminated, as explained in the main text, by setting : $a_{n>N}=a_{\infty}$ and $b_{n>N}=b_{\infty}$ with $a_{\infty}=-$ 0.308651 and $b_{\infty}=0.455040$ for our Fletcher parameters.

Table I. - a) First and fourth neighbours $(R=1$ and 4$)$; in order to simplify the notations we write $: G_{\lambda \mu}^{1(4)}=G_{\lambda \mu}$ $\varepsilon_{i}= \pm 1$.

\begin{tabular}{|c|c|c|c|}
\hline$\lambda, \mu$ & $\begin{array}{c}\text { neighbour } \\
a \sqrt{R(1,0,0)}\end{array}$ & $\begin{array}{c}\text { neighbour } \\
a \sqrt{R(0,1,0)}\end{array}$ & $\begin{array}{c}\text { neighbour } \\
a \sqrt{R}\left(\varepsilon_{1}, \varepsilon_{2}, \varepsilon_{3}\right) / 2\end{array}$ \\
\hline $\begin{array}{l}1,1 \\
1,2 \\
1,3 \\
1,4 \\
1,5 \\
2,1 \\
2,2 \\
2,3 \\
2,4 \\
2,5 \\
3,1 \\
3,2 \\
3,3 \\
3,4 \\
3,5 \\
4,1 \\
4,2 \\
4,3 \\
4,4 \\
4,5 \\
5,1 \\
5,2 \\
5,3 \\
5,4 \\
5,5\end{array}$ & $\begin{array}{l}G_{11} \\
0 \\
0 \\
0 \\
0 \\
0 \\
G_{22} \\
0 \\
0 \\
0 \\
0 \\
0 \\
G_{33} \\
0 \\
0 \\
0 \\
0 \\
0 \\
G_{44} \\
G_{45} \\
0 \\
0 \\
0 \\
G_{45} \\
G_{55}\end{array}$ & $\begin{array}{l}G_{11} \\
0 \\
0 \\
0 \\
0 \\
0 \\
G_{33} \\
0 \\
0 \\
0 \\
0 \\
0 \\
G_{22} \\
0 \\
0 \\
0 \\
0 \\
0 \\
G_{44} \\
-G_{45} \\
0 \\
0 \\
0 \\
-G_{45} \\
G_{55}\end{array}$ & $\begin{array}{l}\left(G_{11}+3 G_{55}\right) / 4 \\
-\varepsilon_{1} \varepsilon_{3} \sqrt{3 / 8} G_{45} \\
-\varepsilon_{2} \varepsilon_{3} \sqrt{3 / 8} G_{45} \\
0 \\
\varepsilon_{1} \varepsilon_{2} \sqrt{3}\left(G_{55}-G_{11}\right) / 4 \\
-\varepsilon_{1} \varepsilon_{3} \sqrt{3 / 8} G_{45} \\
\left(G_{22}+G_{33}+2 G_{44}\right) / 4 \\
\varepsilon_{1} \varepsilon_{2}\left(2 G_{44}-G_{22}-G_{33}\right) / 4 \\
\varepsilon_{2} \varepsilon_{3}\left(G_{22}-G_{33}\right) / \sqrt{8} \\
-\varepsilon_{2} \varepsilon_{3} G_{45} / \sqrt{8} \\
-\varepsilon_{2} \varepsilon_{3} \sqrt{3 / 8} G_{45} \\
\varepsilon_{1} \varepsilon_{2}\left(2 G_{44}-G_{22}-G_{33}\right) / 4 \\
\left(G_{22}+G_{33}+2 G_{44}\right) / 4 \\
\varepsilon_{1} \varepsilon_{3}\left(G_{33}-G_{22}\right) / \sqrt{8} \\
-\varepsilon_{1} \varepsilon_{3} G_{45} / \sqrt{8} \\
0 \\
\varepsilon_{2} \varepsilon_{3}\left(G_{22}-G_{33}\right) / \sqrt{8} \\
\varepsilon_{1} \varepsilon_{3}\left(G_{33}-G_{22}\right) / \sqrt{8} \\
\left(G_{22}+G_{33}\right) / 2 \\
0 \\
\varepsilon_{1} \varepsilon_{2} \sqrt{3}\left(G_{55}-G_{11}\right) / 4 \\
-\varepsilon_{2} \varepsilon_{3} G_{45} / \sqrt{8} \\
-\varepsilon_{1} \varepsilon_{3} G_{45} / \sqrt{8} \\
0 \\
\left(3 G_{11}+G_{55}\right) / 4 \\
\end{array}$ \\
\hline
\end{tabular}


b) Second neighbours $(R=2)$ : in order to simplify the notations we write $\widetilde{G}_{\lambda \mu}^{2}=G_{\lambda \mu} \varepsilon_{i}= \pm 1, \varepsilon= \pm 1$.

\begin{tabular}{|l|l|l|}
\hline$\lambda, \mu$ & neighbour $a\left(\varepsilon_{1}, \varepsilon_{2}, 0\right)$ & \multicolumn{1}{c|}{ neighbour $a(0,0, \varepsilon)$} \\
\hline 1,1 & $G_{11}$ & $G_{22}-G_{23}^{:}$ \\
1,2 & 0 & 0 \\
1,3 & 0 & 0 \\
1,4 & 0 & 0 \\
1,5 & $\varepsilon_{1} \varepsilon_{2} G_{15}$ & 0 \\
2,1 & 0 & 0 \\
2,2 & $G_{22}$ & $G_{22}+G_{23}$ \\
2,3 & $\varepsilon_{1} \varepsilon_{2} G_{23}$ & 0 \\
2,4 & 0 & 0 \\
2,5 & 0 & 0 \\
3,1 & 0 & 0 \\
3,2 & $\varepsilon_{1} \varepsilon_{2} G_{23}$ & 0 \\
3,3 & $G_{22}$ & $G_{44}$ \\
3,4 & 0 & 0 \\
3,5 & 0 & 0 \\
4,1 & 0 & 0 \\
4,2 & 0 & 0 \\
4,3 & 0 & 0 \\
4,4 & $G_{44}$ & $\left(G_{11}+3 G_{55}+\sqrt{12} G_{15}\right) / 4$ \\
4,5 & 0 & $\left(G_{55} \sqrt{3}-G_{11} \sqrt{3}-2 G_{15}\right) / 4$ \\
5,1 & $\varepsilon_{1} \varepsilon_{2} G_{15}$ & 0 \\
5,2 & 0 & 0 \\
5,3 & 0 & 0 \\
5,4 & 0 & $\left(G_{55} \sqrt{3}-G_{11} \sqrt{3}-2 G_{15}\right) / 4$ \\
5,5 & $G_{55}$ & $\left(3 G_{11}+G_{55}-\sqrt{12} G_{15}\right) / 4$ \\
\hline
\end{tabular}


c) Third neighbours $(R=3)$ : in order to simplify the notations we write $\widetilde{G}_{\lambda \mu}^{3}=G_{\lambda \mu} \varepsilon_{i}= \pm 1$.

\begin{tabular}{|c|c|c|c|c|}
\hline$\lambda, \mu$ & $\begin{array}{l}\text { neighbour } \\
a\left(\varepsilon_{1}, 0, \varepsilon_{3}\right)\end{array}$ & $\begin{array}{l}\text { neighbour } \\
a\left(0, \varepsilon_{2}, \varepsilon_{3}\right)\end{array}$ & $\begin{array}{c}\text { neighbour } \\
a\left(\varepsilon_{1}, 3 \varepsilon_{2}, \varepsilon_{3}\right) / 2\end{array}$ & $\begin{array}{c}\text { neighbour } \\
a\left(3 \varepsilon_{2}, \varepsilon_{1}, \varepsilon_{3}\right) / 2\end{array}$ \\
\hline $\begin{array}{l}1,1 \\
1,2 \\
1,3 \\
1,4 \\
1,5 \\
2,1 \\
2,2 \\
2,3 \\
2,4 \\
2,5 \\
3,1 \\
3,2 \\
3,3 \\
3,4 \\
3,5 \\
4,1 \\
4,2 \\
4,3 \\
4,4 \\
4,5 \\
5,1 \\
5,2 \\
5,3 \\
5,4 \\
5,5\end{array}$ & $\begin{array}{l}G_{11} \\
\varepsilon_{1} \varepsilon_{3} G_{12} \\
0 \\
0 \\
0 \\
\varepsilon_{1} \varepsilon_{3} G_{12} \\
G_{22} \\
0 \\
0 \\
0 \\
0 \\
0 \\
G_{33} \\
\varepsilon_{1} \varepsilon_{3} G_{34} \\
\varepsilon_{1} \varepsilon_{3} G_{35} \\
0 \\
0 \\
\varepsilon_{1} \varepsilon_{3} G_{34} \\
G_{44} \\
G_{45} \\
0 \\
0 \\
\varepsilon_{1} \varepsilon_{3} G_{35} \\
G_{45} \\
G_{55}\end{array}$ & $\begin{array}{l}G_{11} \\
0 \\
\varepsilon_{2} \varepsilon_{3} G_{12} \\
0 \\
0 \\
0 \\
G_{33} \\
0 \\
-\varepsilon_{2} \varepsilon_{3} G_{34} \\
\varepsilon_{2} \varepsilon_{3} G_{35} \\
\varepsilon_{2} \varepsilon_{3} G_{12} \\
0 \\
G_{22} \\
0 \\
0 \\
0 \\
-\varepsilon_{2} \varepsilon_{3} G_{34} \\
0 \\
G_{44} \\
-G_{45} \\
0 \\
\varepsilon_{2} \varepsilon_{3} G_{35} \\
0 \\
-G_{45} \\
G_{55}\end{array}$ & $\begin{array}{l}\left(G_{11}+3 G_{55}\right) / 4 \\
\varepsilon_{1} \varepsilon_{3}\left(G_{12}+G_{35} \sqrt{3}+G_{45} \sqrt{6}\right) / 4 \\
\varepsilon_{2} \varepsilon_{3}\left(G_{12}+G_{35} \sqrt{3}-G_{45} \sqrt{6}\right) / 4 \\
\varepsilon_{1} \varepsilon_{2}\left(G_{12}-G_{35} \sqrt{3}\right) / \sqrt{8} \\
\varepsilon_{1} \varepsilon_{2}\left(G_{11}-G_{55}\right) \sqrt{3} / 4 \\
\varepsilon_{1} \varepsilon_{3}\left(G_{12}+G_{35} \sqrt{3}+G_{45} \sqrt{6}\right) / 4 \\
\left(G_{22}+G_{33}+2 G_{44}+G_{34} \sqrt{8}\right) / 4 \\
\varepsilon_{1} \varepsilon_{2}\left(G_{22}+G_{33}-2 G_{44}\right) / 4 \\
\varepsilon_{2} \varepsilon_{3}\left(G_{22}-G_{33}-G_{34} \sqrt{2}\right) / \sqrt{8} \\
\varepsilon_{2} \varepsilon_{3}\left(G_{12} \sqrt{3}-G_{35}-G_{45} \sqrt{2}\right) / 4 \\
\varepsilon_{2} \varepsilon_{3}\left(G_{12}+G_{35} \sqrt{3}-G_{45} \sqrt{6}\right) / 4 \\
\varepsilon_{1} \varepsilon_{2}\left(G_{22}+G_{33}-2 G_{44}\right) / 4 \\
\left(G_{22}+G_{33}+2 G_{44}-G_{34} \sqrt{8}\right) / 4 \\
\varepsilon_{1} \varepsilon_{3}\left(G_{22}-G_{33}+G_{34} \sqrt{2}\right) / \sqrt{8} \\
\varepsilon_{1} \varepsilon_{3}\left(G_{12} \sqrt{3}-G_{35}+G_{45} \sqrt{2}\right) / 4 \\
\varepsilon_{1} \varepsilon_{2}\left(G_{12}-G_{35} \sqrt{3}\right) / \sqrt{8} \\
\varepsilon_{2} \varepsilon_{3}\left(G_{22}-G_{33}-G_{34} \sqrt{2}\right) / \sqrt{8} \\
\varepsilon_{1} \varepsilon_{3}\left(G_{22}-G_{33}+G_{34} \sqrt{2}\right) / \sqrt{8} \\
\left(G_{22}+2 G_{33}\right) / 4 \\
\left(G_{12} \sqrt{3}+G_{35}\right) / \sqrt{8} \\
\varepsilon_{1} \varepsilon_{2}\left(G_{11}-G_{55}\right) \sqrt{3} / 4 \\
\varepsilon_{2} \varepsilon_{3}\left(G_{12} \sqrt{3}-G_{35}-G_{45} \sqrt{2}\right) / 4 \\
\varepsilon_{1} \varepsilon_{3}\left(G_{12} \sqrt{3}-G_{35}+G_{45} \sqrt{2}\right) / 4 \\
\left(G_{12} \sqrt{3}+G_{35}\right) / \sqrt{8} \\
\left(3 G_{11}+G_{55}\right) / 4\end{array}$ & $\begin{array}{l}\left(G_{11}+3 G_{55}\right) / 4 \\
\varepsilon_{2} \varepsilon_{3}\left(G_{12}+G_{35} \sqrt{3}-G_{45} \sqrt{6}\right) / 4 \\
\varepsilon_{1} \varepsilon_{3}\left(G_{12}+G_{35} \sqrt{3}+G_{45} \sqrt{6}\right) / 4 \\
\varepsilon_{1} \varepsilon_{2}\left(G_{35} \sqrt{3}-G_{12}\right) / \sqrt{8} \\
\varepsilon_{1} \varepsilon_{2}\left(G_{11}-G_{55}\right) \sqrt{3} / 4 \\
\varepsilon_{2} \varepsilon_{3}\left(G_{12}+G_{35} \sqrt{3}-G_{45} \sqrt{6}\right) / 4 \\
\left(G_{22}+G_{33}+2 G_{44}-G_{34} \sqrt{8}\right) / 4 \\
\varepsilon_{1} \varepsilon_{2}\left(G_{22}+G_{33}-2 G_{44}\right) / 4 \\
\varepsilon_{1} \varepsilon_{3}\left(G_{33}-G_{22}-G_{34} \sqrt{2}\right) / \sqrt{8} \\
\varepsilon_{1} \varepsilon_{3}\left(G_{12} \sqrt{3}-G_{35}+G_{45} \sqrt{2}\right) / 4 \\
\varepsilon_{1} \varepsilon_{3}\left(G_{12}+G_{35} \sqrt{3}+G_{45} \sqrt{6}\right) / 4 \\
\varepsilon_{1} \varepsilon_{2}\left(G_{22}+G_{33}-2 G_{44}\right) / 4 \\
\left(G_{22}+G_{33}+2 G_{44}+G_{34} \sqrt{8}\right) / 4 \\
\varepsilon_{2} \varepsilon_{3}\left(G_{33}-G_{22}+G_{34} \sqrt{2}\right) / \sqrt{8} \\
\varepsilon_{2} \varepsilon_{3}\left(G_{12} \sqrt{3}-G_{35}-G_{45} \sqrt{2}\right) / 4 \\
\varepsilon_{1} \varepsilon_{2}\left(G_{35} \sqrt{3}-G_{12}\right) / \sqrt{8} \\
\varepsilon_{1} \varepsilon_{3}\left(G_{33}-G_{22}-G_{34} \sqrt{2}\right) / \sqrt{8} \\
\varepsilon_{2} \varepsilon_{3}\left(G_{33}-G_{22}+G_{34} \sqrt{2}\right) / \sqrt{8} \\
\left(G_{22}+2 G_{33}\right) / 4 \\
-\left(G_{12} \sqrt{3}+G_{35}\right) / \sqrt{8} \\
\varepsilon_{1} \varepsilon_{2}\left(G_{11}-G_{55}\right) \sqrt{3} / 4 \\
\varepsilon_{1} \varepsilon_{3}\left(G_{12} \sqrt{3}-G_{35}+G_{45} \sqrt{2}\right) / 4 \\
\varepsilon_{2} \varepsilon_{3}\left(G_{12} \sqrt{3}-G_{35}-G_{45} \sqrt{2}\right) / 4 \\
-\left(G_{12} \sqrt{3}+G_{35}\right) / \sqrt{8} \\
\left(3 G_{11}+G_{55}\right) / 4\end{array}$ \\
\hline
\end{tabular}

Table II. - Compilation of the exact coefficients (Eq. 1) that are required to compute the independent $\tilde{G}_{\lambda \mu}^{R}$ of table $I$ in terms of the intra-atomic $G_{\lambda \lambda}^{00}, G_{\mu \mu}^{00}$ and $G_{\lambda \mu}^{0 R}(E q .3)$, for the FCC structure, up to the 4 th neighbour. The hopping integrals are defined in reference $[12]\left(W_{0}=2.7 \mathrm{eV}\right)$.
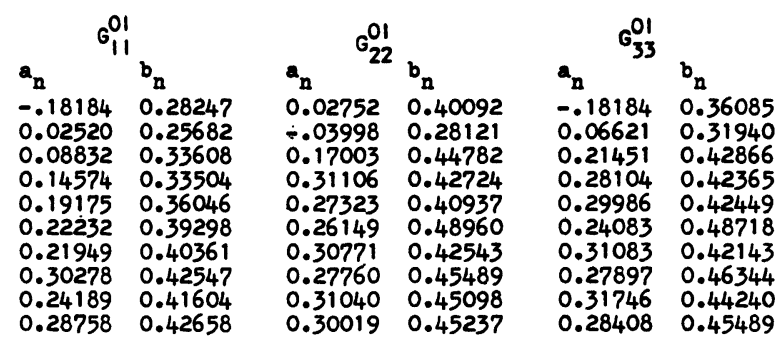

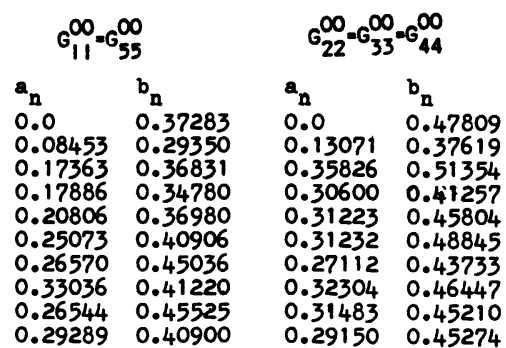

\begin{tabular}{ll}
\multicolumn{3}{c}{$G_{55}^{01}$} \\
$a_{n}$ & $b_{n}$ \\
0.10505 & 0.34856 \\
0.08471 & 0.29584 \\
0.22065 & 0.35450 \\
0.16770 & 0.34980 \\
0.22547 & 0.36597 \\
0.26721 & 0.39807 \\
0.30230 & 0.44785 \\
0.31375 & 0.41167 \\
0.29227 & 0.44169 \\
0.28088 & 0.42122
\end{tabular}

$0.28088 \quad 0.42122$

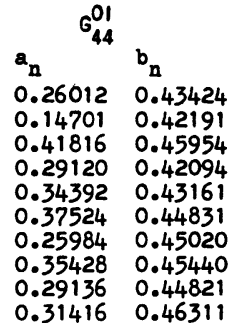

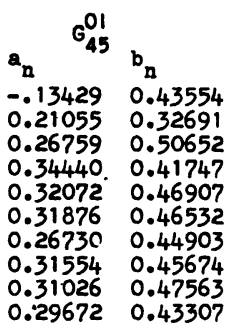


Table II (continued).
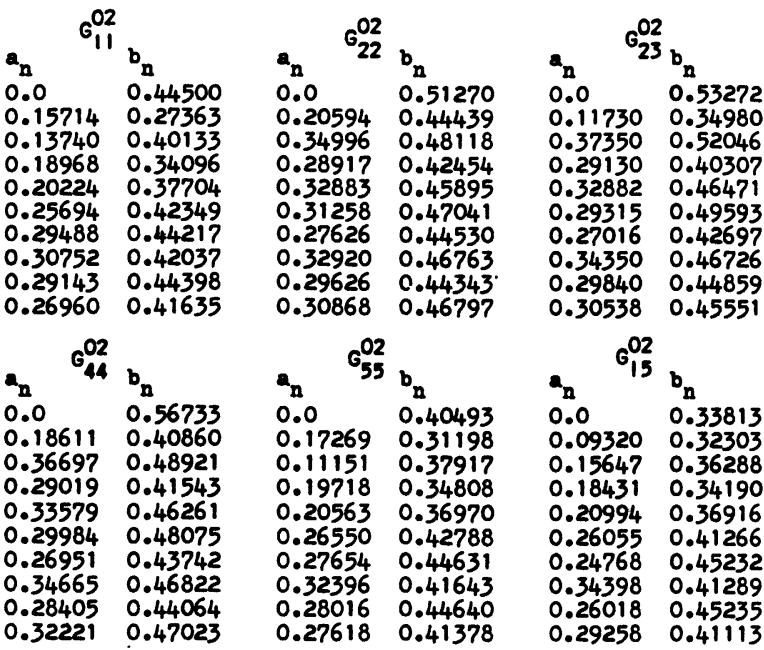

0.172690 .31198

$\begin{array}{ll}0.11151 & 0.37917 \\ 0.19718 & 0.34808\end{array}$

$\begin{array}{ll}0.19718 & 0.34808 \\ 0.20563 & 0.36970\end{array}$

$\begin{array}{ll}0.20563 & 0.36970 \\ 0.26550 & 0.42788\end{array}$

$\begin{array}{ll}0.26550 & 0.42788 \\ 0.27654 & 0.44631\end{array}$

$\begin{array}{ll}0.27654 & 0.44631 \\ 0.32396 & 0.41643\end{array}$

$\begin{array}{ll}0.28016 & 0.44640 \\ 0.27618 & 0.41378\end{array}$
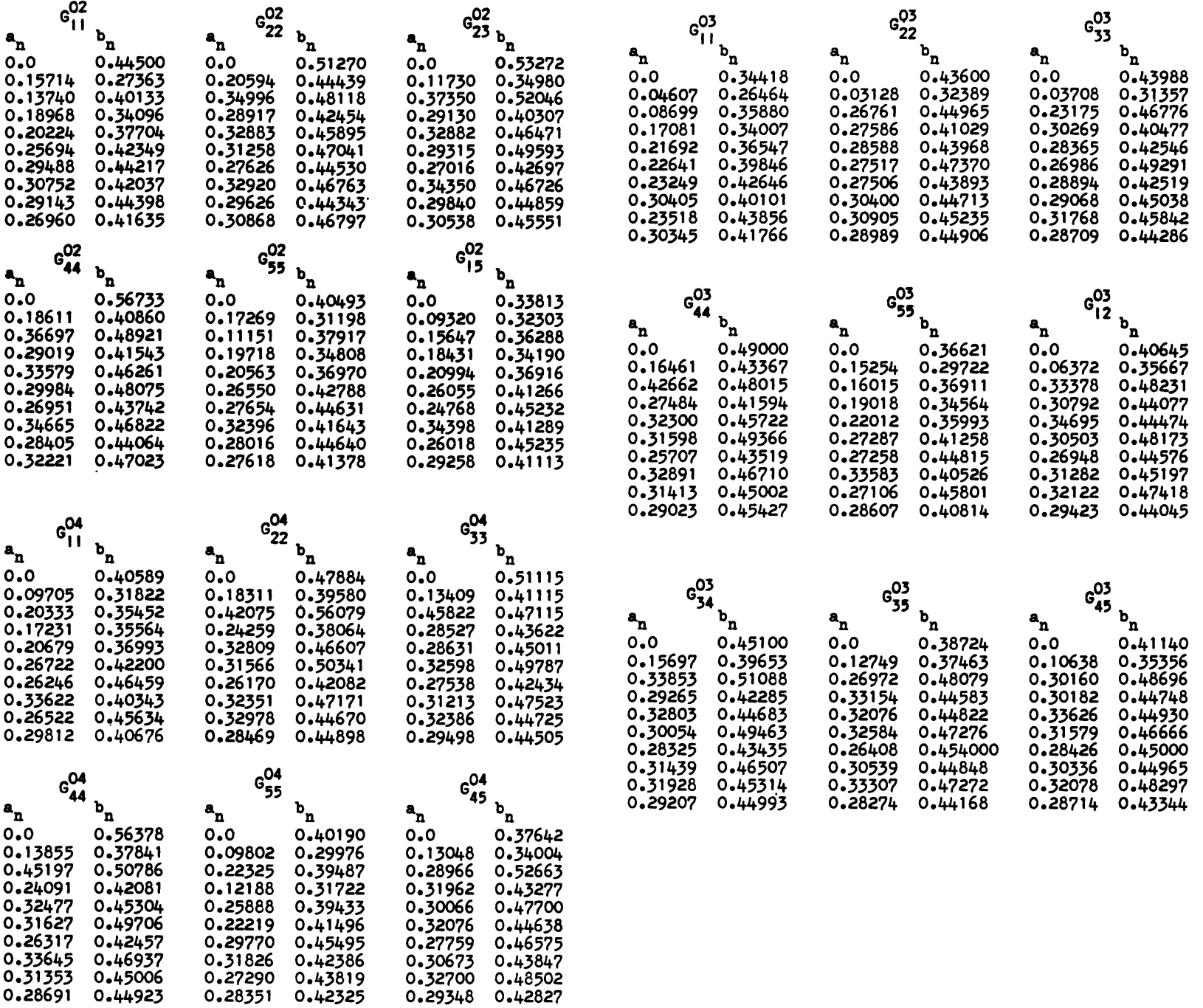

\begin{tabular}{ll}
\multicolumn{3}{c}{$G_{55}^{04}$} \\
$a_{n}$ & $b_{n}$ \\
0.0 & 0.40190 \\
0.09802 & 0.29976 \\
0.22325 & 0.39487 \\
0.12188 & 0.31722 \\
0.25888 & 0.39433 \\
0.22219 & 0.41496 \\
0.29770 & 0.45495 \\
0.31826 & 0.42386 \\
0.27290 & 0.43819 \\
0.28351 & 0.42325
\end{tabular}
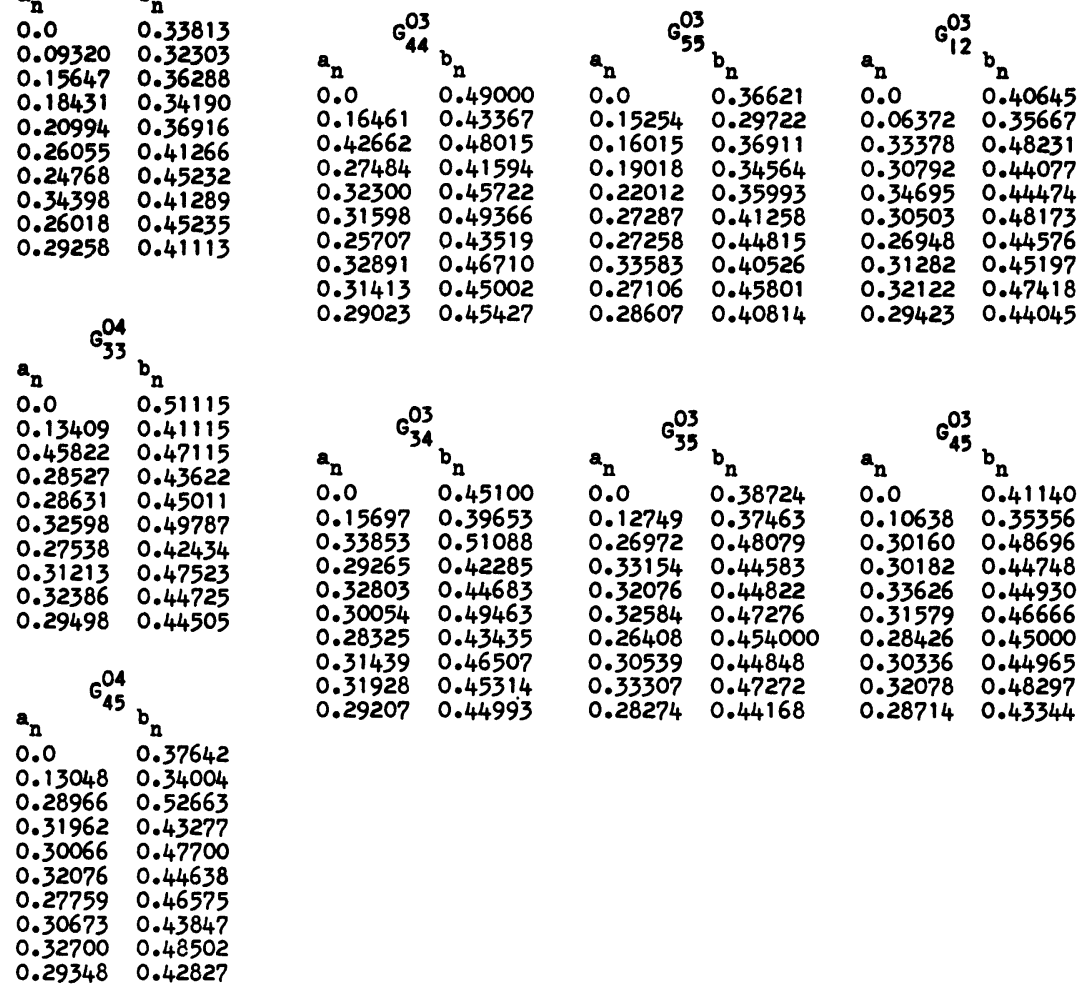

$\begin{array}{ll}0.31928 & 0.45314 \\ 0.29207 & 0.44993\end{array}$

0.282740 .44168

$\begin{array}{ll}0.28714 & 0.43344\end{array}$

\section{References}

[1] Haydock, R., Heine, V. and Kelly, M. J., J. Phys. C 5 (1972) 2845 ; J. Phys. C 8 (1975) 2591.

[2] Haydock, R., Solid State Phys. 35 (1980) 216.

[3] Kelly, M. J., Solid State Phys. 35 (1980) 296.

[4] Tréglia, G., Ducastelle, F. and SpanjaArd, D., J. Physique 41 (1980) 281.

[5] Bieber, A., Gautier, F., Tréglia, G. and DucasTELLE, F., Solid State Commun. 39 (1981) 149.

[6] Lehmann, G. and TaUt, M., Phys. Status Solidi (b) 54 (1972) 469.

[7] Gaspard, J. P. and Cyrot-Lackmann, F., J. Phys. C 6 (1973) 3077.

[8] Ehrenreich, H. and Hodges, L., Methods Comput. Phys. 8 (1968) 149.

[9] Ducastelle, F., J. Physique 31 (1970) 1055.

[10] Turchi, P., Ducastelle, F. and Trégla, G., J. Phys. C 15 (1982) 2891.
[11] Koenig, C., Z. Phys. B 50 (1983) 33.

[12] Fletcher, G. C., Proc. Phys. Soc. A 267 (1954) 505.

[13] Bieber, A., Ducastelle, F., Gautier, F., Tréglia, G. and TurChI, P., Solid State Commun. 45 (1983) 585.

[14] Dieudonné, J., Calcul Infinitésimal (ed. Hermann Paris) 1968, 162.

[15] Hirai, K. and Kanamori, J., J. Phys. Soc. Japan 50 (1981) 2265

[16] Bieber, A. and Gautier, F., Proc. 12th Ann. Int. Symp. on Electronic Structure of Metals and Alloys, Gaussig (1982) 13 ; and to be published.

[17] Terakura, K., Hamada, N., Ogushi, T. and Asada, T., J. Phys. F 12 (1982) 1661.

[18] Hirai, K., J. Phys. Soc. Japan 51 (1982) 1134. 